\title{
PERSONALITY AND AUGMENTING/REDUCING IN VISUAL AND AUDITORY EVOKED POTENTIALS
}

\author{
G. STEnBerg, ', I. Rosén ${ }^{1}$ and J. RisBerg ${ }^{2}$ \\ Departments of 'Clinical Neurophysiology and 'Psychiatry, University of Lund, S-221 85 Lund, Sweden
}

(Received 18 February 1987; revised 14 July 1987)

\begin{abstract}
Summary-Previous studies have indicated a relationship between evoked potential augmenting/reducing and extraversion or sensation seeking. However, the proposed mechanism of protective inhibition can account for this fact only if the relationship generalizes across different modalities and response definitions. The present study was designed to test this, using six intensities of visual and six intensities of auditory stimuli along with the EPI and SSS questionnaires.

For the visual stimuli, the slope of the P90-N120 amplitude at the vertex correlated significantly with both the extraversion and the disinhibition scales in the way that augmenting/reducing theory predicts.

However, over the primary visual area, no component showed the same personality relationship as the vertex wave, and one early component showed the opposite. This result suggests that personality differences in VEPs may reflect different ways of allocating processing resources between primary and association areas, rather than a generalized tendency to inhibit strong stimuli.

In the auditory modality, personality differences were not apparent in the amplitude slopes, possibly due to the confluence from primary and association areas in AEPs in the vertex lead.

There was a general tendency for latencies to correlate positively with extraversion and disinhibition, in congruence with Eysenck's theory on the biological basis of extraversion.
\end{abstract}

\section{INTRODUCTION}

The relation between evoked potential amplitude and stimulus intensity is known to vary between subjects, and at least some of this interindividual variability can be ascribed to personality differences.

Thus, a pattern of amplitude increases across intensities has been found in a group of people, referred to as augmenters, whereas their counterparts, the reducers, reach their maximum amplitudes at lower stimulus intensities. Augmenting has been associated with the personality traits of extraversion (Soskis and Shagass, 1974; Friedman and Meares, 1979) and sensation seekingespecially disinhibition - (Zuckerman, Murtaugh and Siegel, 1974; von Knorring, 1980) and, conversely, reducing with introversion and sensation avoiding. This view has come to prevail, although it has met some conflicting findings, ranging from the opposite (Haier, Robinson, Braden and Williams, 1984) to the somewhat dissonant, such as higher pain sensitivity in augmenters (von Knorring, 1974).

As explanation, it has been suggested (Buchsbaum and Silverman, 1968) that a mechanism for regulation of the sensory input is at work in reducers, analogous to, or identical with, Pavlovian protective inhibition. At high stimulus intensities, reducing is assumed to avert the threat of sensory overload by dampening the impact of the stimuli. To the extent that reducing is linked to introversion and sensation avoiding, protective inhibition would act in concord with a withdrawn lifestyle to provide a double protection.

However, the implications of this hypothesis reach farther than the empirical findings have been able to follow. Thus, unless subjective perception can be identified with a single EP component at a particular electrode site, the augmenting/reducing tendency ought to generalize across sites and components. Evidence for this has been sparse. An informal tradition has been established, in which augmenting/reducing is measured as the slope of the vertex P100-N120 amplitude regressed on $\log$ intensity (e.g. Haier et al., 1984). Other response definitions have been tried, but they have not been proven equivalent to this alternative. [Different measures have been examined critically by Connolly and Gruzelier (1982)]. 
Similarly, in as far as stimuli from all modalities can be disturbing at high intensities, the protective inhibitory mechanism ought to safeguard against them all. However, outside of the favoured visual modality, demonstrations of augmenting/reducing have been few (Mullins and Lukas, 1984; Como, Simons and Zuckerman, 1984) and cross-modal correlations have fallen short of significance (Buchsbaum, Haier and Johnson, 1983; Kaskey, Salzman, Klorman and Pass, 1980; Raine, Mitchell and Venables, 1981).

This study was undertaken to investigate whether relations could be established between personality variables and augmenting/reducing in the visual and auditory modalities. Furthermore, the object was to compare different response definitions in each modality with the traditional one.

As personality variables, extraversion and neuroticism from the Eysenck Personality Inventory were used, along with the disinhibition subscale from the Zuckerman Sensation Seeking Scale (Eysenck and Eysenck, 1970; Zuckerman, 1979).

\section{METHOD}

\section{Subjects}

Thirty-one psychology students volunteered and were paid for their participation. One subject was excluded from data analysis due to artifacts in the EP recording. Of the remaining 30 subjects, 22 were female. Age ranged from 18 to 42 , with an average of 24 .

\section{Personality measurement}

All subjects completed the Eysenck Personality Inventory, form A, and the Zuckerman Sensation Seeking Scale, form IV (both in Swedish translations); one before and the other after the evoked potential recording. Intercorrelations between personality scales are given in Table 1.

\section{Procedure}

Evoked potential recording was made in a darkened room, where the subject sat in a chair with a head rest, wearing headphones and facing a screen behind which the photostimulator was mounted. Instructions for the visual EPs were to fixate a point on the centre of the screen and to watch the flashes while trying to avoid blinking immediately following a flash. For the auditory stimuli, the subject was instructed to listen to the tones with closed eyes.

Six intensities of flash-light and six intensities of sine-wave tones were used. Each stimulus was presented 64 times separated by an interval randomized around $2 \mathrm{sec}$. After each block of stimulus presentations there was a rest period of $2 \mathrm{~min}$ before the next.

Visual and auditory stimuli were alternated, the presentation order within each modality being randomized and kept constant for all subjects.

Flashes were produced by a Siemens photo stimulator with three settings. Additional intensity levels were obtained by interposing plates of frosted glass between the stimulator and the translucent screen on which the flashes were back projected. The size of the screen was $30 \times 45 \mathrm{~cm}$ and it was placed $110 \mathrm{~cm}$ in front of the subject.

Light intensity was measured from the place of the subject. Due to the brief duration of the flashes $(40 \mu \mathrm{sec})$, peak intensity could not be captured by available luminance meters. Instead, the

Table 1. Correlations between personality variables (from EPI: extraversion, neuroticism and lie. From SSS: general sensation seeking, thrill and adventure seeking, experience seeking, disinhibition and boredom susceptibility)

\begin{tabular}{lccccccr}
\hline & $\mathrm{N}$ & $\mathrm{L}$ & GEN & TAS & \multicolumn{1}{c}{ ES } & \multicolumn{1}{c}{ DIS } & \multicolumn{1}{c}{ BS } \\
\hline E & 0.05 & -0.31 & 0.38 & 0.46 & 0.20 & 0.55 & 0.18 \\
N & & -0.08 & 0.11 & 0.15 & 0.26 & -0.10 & -0.07 \\
L & & & 0.05 & 0.01 & -0.10 & -0.25 & -0.21 \\
GEN & & & & 0.84 & 0.79 & 0.70 & 0.59 \\
TAS & & & & & 0.68 & 0.62 & 0.33 \\
ES & & & & & & 0.51 & 0.41 \\
DIS & & & & & & & 0.48 \\
\hline
\end{tabular}

Table 2. Means and SDs of extraversion scores for groups used in analysis of variance

\begin{tabular}{|c|c|c|c|c|c|c|}
\hline & \multicolumn{3}{|c|}{ Low extraversion } & \multicolumn{3}{|c|}{ High extraversion } \\
\hline & $\mathbf{N}$ & $\mathbf{M}$ & $\mathrm{SD}$ & $\mathbf{N}$ & $\mathbf{M}$ & SD \\
\hline Both sexes & 15 & 9.47 & 2.42 & 15 & 16.33 & 2.09 \\
\hline Males & 3 & 10.00 & 2.65 & 5 & 16.80 & 1.79 \\
\hline Females & 12 & 9.33 & 2.46 & 10 & 16.10 & 2.28 \\
\hline
\end{tabular}


stimulator was set blinking at the maximum rate of $40 \mathrm{~Hz}$, and the resulting intermittent light was measured. In this way, relative magnitude is preserved, but actual momentary intensity is underestimated. The values obtained were $10,22,39,79,175$ and $295 \mathrm{~cd} \mathrm{~m}^{-2}$, corresponding to $2.9,6.4,11.3,22.9,50.8$ and $85.6 \mathrm{ft}-\mathrm{L}$. Logarithms of light intensities were used in the calculation of amplitude/intensity slopes.

Sound stimuli were delivered through headphones for a duration of $200 \mathrm{msec}$ each, with rise and fall times of $20 \mathrm{msec}$ to avoid click phenomena. The tones had a frequency of $1000 \mathrm{~Hz}$, and their intensities were 50,60,70,80,90 and $100 \mathrm{~dB}$ HL. (The intensities are given in $\mathrm{dB}$ HL as calibrated on the audiometer which delivered the tones, i.e. hearing levels were not individually determined. Since individual sensitivity to low-intensity stimulation is known to co-vary to some extent with extraversion-introversion, correcting for this sensitivity might have removed some of the variance of interest for this study.)

EEG was recorded with tin-plate electrodes applied with bentonite paste at $\mathrm{Fz}, \mathrm{Cz}$ and $\mathrm{Oz}$, all referred to the left mastoid using $\mathrm{Pz}$ as ground. The $\mathrm{Cz}$ and $\mathrm{Oz}$ leads were used in the visual EPs; $\mathrm{Fz}$ and $\mathrm{Cz}$ in the auditory. An automatic artifact rejection procedure was used, discarding sweeps containing voltages in excess of $200 \mu \mathrm{V}$ on any channel. Electrode impedances were kept below $10 \mathrm{k} \Omega$.

The filter pass band was $0.5-200 \mathrm{~Hz}$. Sampling was done at $2048 \mathrm{~Hz}$ during the $100 \mathrm{msec}$ preceding the stimulus and the $400 \mathrm{msec}$ following it, and the averaged EPs were stored in digitized form on disk. Later, they were digitally filtered with a low-pass setting of $70 \mathrm{~Hz}$, and the prestimulus epoch was averaged to form a baseline which was then subtracted from all subsequent data points.

Peaks were identified and measured as to amplitude and latency by a computer program. The algorithm accepted as peaks only points where the first derivative crossed zero. To ensure consistency of peak identification, each subject's curves were first averaged across intensities, and the peak latencies found on this average curve were made the center of the time windows in which peak identification for the individual curves took place. The width of the time window was set to $\pm 1 \mathrm{SD}$ of the latency distribution for the peak found in a preliminary study. If no point was found to satisfy the criterion in the first iteration, the process was repeated using a wider time window until a band of \pm 3 SDs had been searched. The operator was given the option of overriding the computer's choice at any stage in favour of peak definition by cursor setting on the graphical display. This proved necessary in only a small number of cases. Average latencies for all peaks are given in Tables 3-6. The most extreme latencies found in the visual EPs were as follows: Cz: P90: 55-130, N120: 86-150, P200: 149-245; Oz: N60: 40-103, P100: 89-168, N150: 103-197, P200: $164-250$.

Table 3. Visual stimuli. Site: $\mathrm{Cz}$

\begin{tabular}{|c|c|c|c|c|c|c|}
\hline Component & Average & SD & E-corr. & N-corr. & Dis-corr. & AR-corr. \\
\hline \multicolumn{7}{|l|}{ P90 } \\
\hline Mean amplitude & 0.83 & 3.01 & 0.27 & 0.22 & 0.06 & -0.26 \\
\hline Mean latency & 89.92 & 9.22 & 0.11 & -0.14 & 0.08 & -0.14 \\
\hline Amplitude slope & 0.98 & 1.84 & 0.07 & 0.26 & -0.04 & 0.32 \\
\hline Latency slope & -1.35 & 4.67 & 0.07 & -0.02 & 0.26 & -0.17 \\
\hline \multicolumn{7}{|l|}{ N120 } \\
\hline Mean amplitude & 6.15 & 4.45 & 0.31 & -0.12 & 0.22 & $0.50^{\text {* } ~}$ \\
\hline Mean latency & 116.43 & 10.96 & $0.44^{*}$ & 0.23 & 0.22 & 0.15 \\
\hline Amplitude slope & 0.59 & 2.17 & 0.28 & -0.29 & 0.35 & $0.60^{\text {* }}$ \\
\hline Latency slope & -1.25 & 5.64 & -0.06 & -0.09 & 0.06 & 0.19 \\
\hline \multicolumn{7}{|l|}{ P200 } \\
\hline Mean amplitude & 11.43 & 5.15 & -0.03 & -0.11 & -0.25 & 0.07 \\
\hline Mean latency & 194.52 & 17.55 & 0.30 & 0.12 & 0.03 & 0.13 \\
\hline Amplitude slope & 2.89 & 2.97 & 0.00 & -0.06 & -0.13 & -0.02 \\
\hline Latency slope & -2.27 & 10.89 & 0.26 & 0.07 & 0.24 & 0.34 \\
\hline \multicolumn{7}{|l|}{$\mathrm{P} 90-\mathrm{N} 120$} \\
\hline Mean amplitude & 6.99 & 4.61 & $0.48^{* *}$ & 0.03 & 0.25 & 0.32 \\
\hline Amplitude slope & 1.58 & 1.90 & $0.39^{*}$ & -0.07 & $0.36^{\star}$ & 1.00 \\
\hline \multicolumn{7}{|l|}{ N120-P200 } \\
\hline Mean amplitude & 17.58 & 7.67 & 0.16 & -0.14 & -0.04 & 0.34 \\
\hline Amplitude slope & 3.49 & 3.70 & 0.16 & -0.21 & 0.10 & 0.34 \\
\hline
\end{tabular}

Peak measurements: mean values (averaged over intensities) and slope values (regressed on intensity); their averages, SDs and their correlations with extraversion (E), neuroticism (N), disinhibition (Dis) and the conventional augmenting/reducing-measure (AR) as explained in the text.

For correlations, ${ }^{*} P<0.05, * P<0.01$ (two-tailed probabilities). 
Table 4. Visual stimuli. Site: $O z$

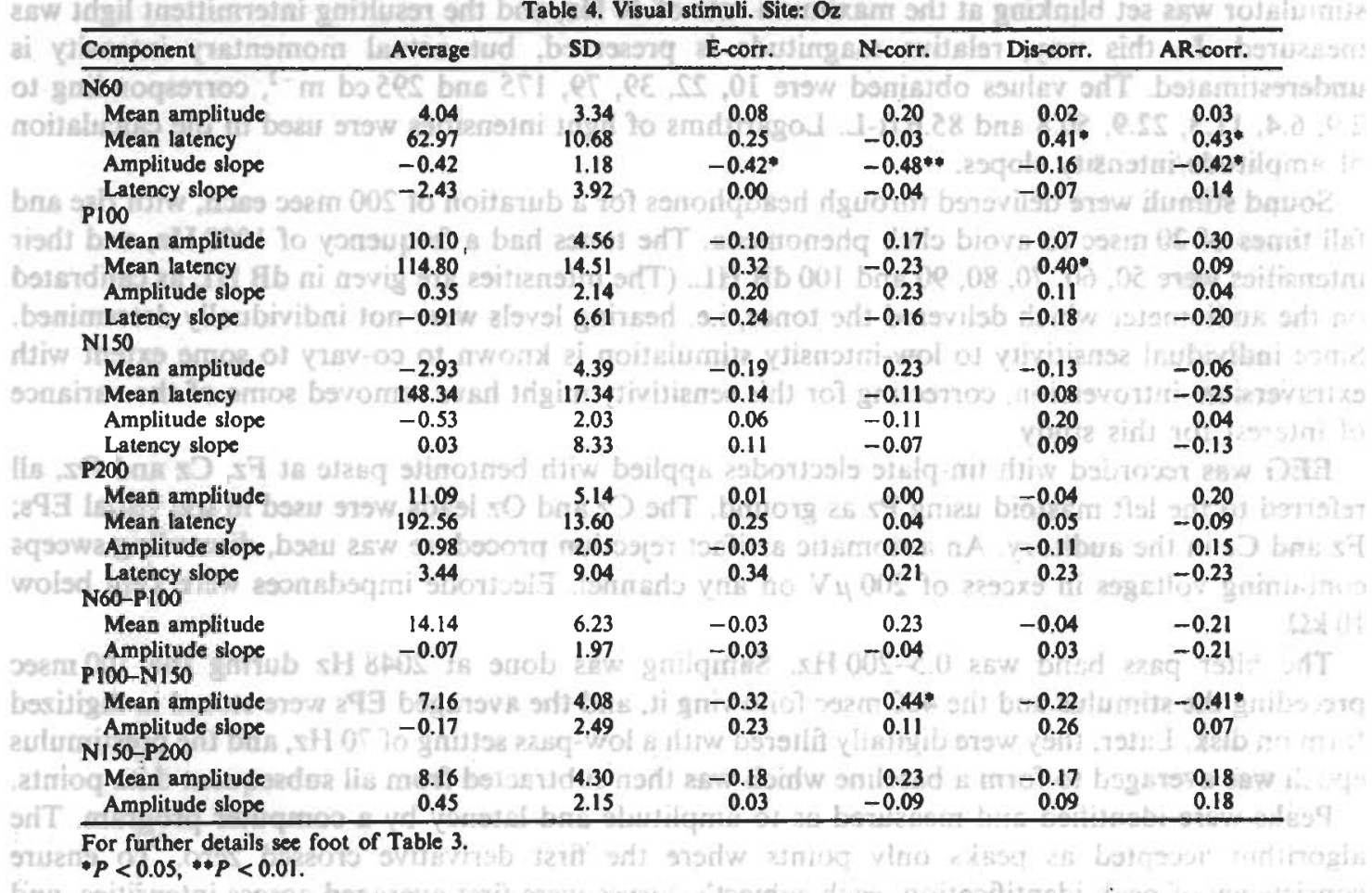

\section{RESULTS}

\section{Grand average curves for personality groups}

Mean curves were calculated for each person by averaging the amplitude at every time point across stimulus intensities. Analogously, slope curves were constructed by regressing the absolute value of the amplitude on intensity at each time point. Disregarding the sign of the amplitude in the latter case ensured that augmenting would show up as positive slope values for positive and negative peaks alike.

Personality groups were formed by splitting the group of subjects at the mean of each personality variable distribution. For each personality group, grand average curves were formed for both the

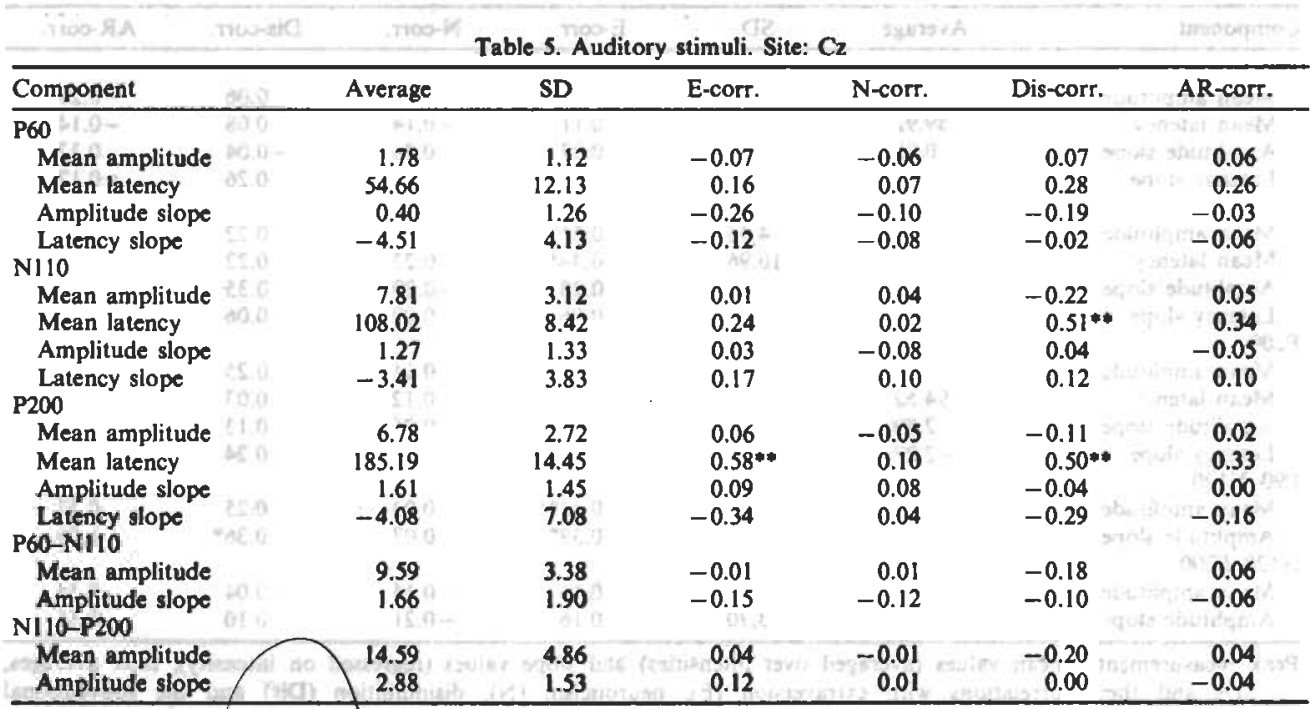

For further details see foot of Table 3 .

* $P<0.05, * * P<0.01$. 
Table 6. Auditory stimuli. Site: Fz

\begin{tabular}{|c|c|c|c|c|c|c|c|}
\hline Component & 17 & Average & SD & E-cors. & N-corr. & Dis-cort. & AR-cort. \\
\hline \multicolumn{8}{|l|}{ P60 } \\
\hline Mean amplitude & & 1.54 & 1.30 & -0.14 & -0.26 & 0.07 & 0.18 \\
\hline Mean latency & & 56.33 & 13.58 & 0.11 & 0.11 & 0.27 & 0.18 \\
\hline Amplitude slope & & 0.11 & 1.23 & -0.15 & -0.24 & -0.17 & 0.14 \\
\hline Latency slope & & -3.27 & 4.07 & -0.42 & 0.02 & -0.23 & -0.16 \\
\hline \multicolumn{8}{|l|}{ N110 } \\
\hline Mean amplitude & & 7.59 & 2.95 & -0.02 & 0.04 & -0.16 & -0.09 \\
\hline Mean latency & & 109.52 & 9.10 & 0.23 & 0.04 & $0.47 * *$ & 0.24 \\
\hline Amplitude slope & & 0.81 & 1.38 & -0.02 & -0.11 & 0.16 & -0.14 \\
\hline \multicolumn{8}{|l|}{ P200 } \\
\hline Mean amplitude & & 4.69 & 2.21 & 0.07 & -0.04 & -0.05 & 0.05 \\
\hline Mean latency & gote & 186.21 & 16.27 & $0.47 * *$ & 0.19 & $0.43^{*}$ & $0.42^{*}$ \\
\hline Amplitude slope & & 0.62 & 1.52 & 0.02 & 0.00 & 0.08 & 0.07 \\
\hline Latency slope & & -4.22 & 5.93 & -0.44 & -0.09 & -0.31 & -0.11 \\
\hline \multicolumn{8}{|l|}{ P60-N110 } \\
\hline Mean amplitude & & 9.13 & 3.09 & -0.08 & -0.07 & -0.12 & -0.01 \\
\hline Amplitude slope & & 0.92 & 1.72 & -0.13 & -0.26 & 0.01 & -0.01 \\
\hline \multicolumn{8}{|l|}{ N110-P200 } \\
\hline Mean amplitude & & 12.27 & expas & 0.02 & 0.01 & -0.14 & -0.04 \\
\hline Amplitude slope & & 1.43 & 1.54 & 0.00 & -0.11 & 0.22 & -0.06 \\
\hline
\end{tabular}

For further details see foot of Table 3.

$* P<0.05, " * P<0.01$.

mean and the slope curves. The results of these comparisons for high vs low extraversion are shown in Figs 1 and 2.

The slope curves indicate that intensity-amplitude increase coincident with major peaks occur more reliably in auditory than in visual EPs. Of the two leads for the visual recording, the one at $\mathrm{Oz}$ shows much less consistent amplitude augmenting of major peaks. At $\mathrm{Cz}$, there is marked

Visual Stimuti

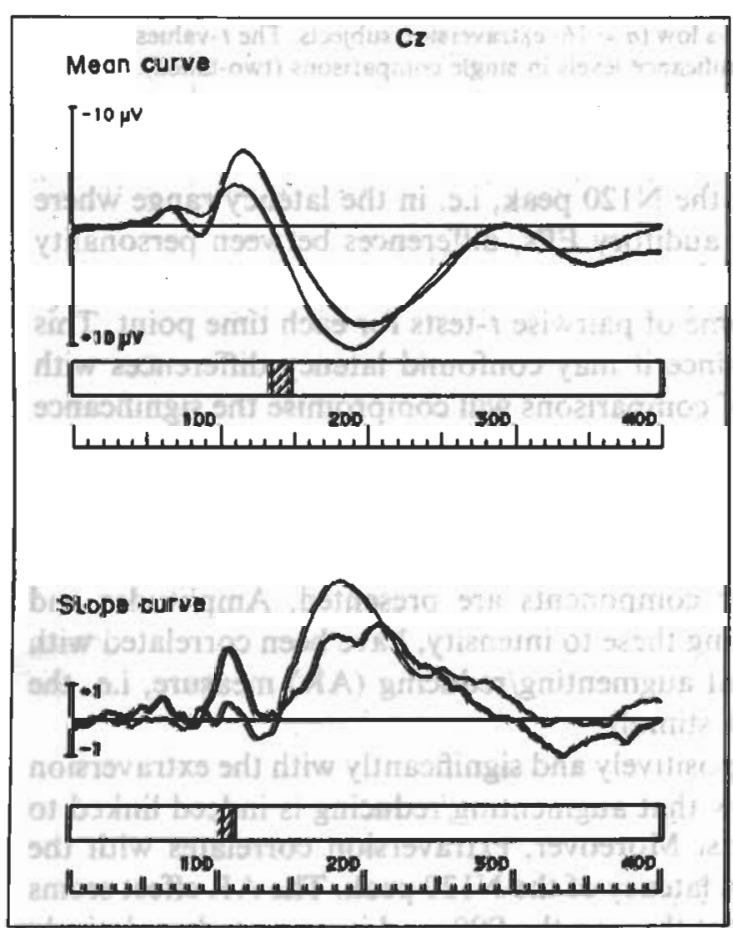

High Extroversion Low Extraversion

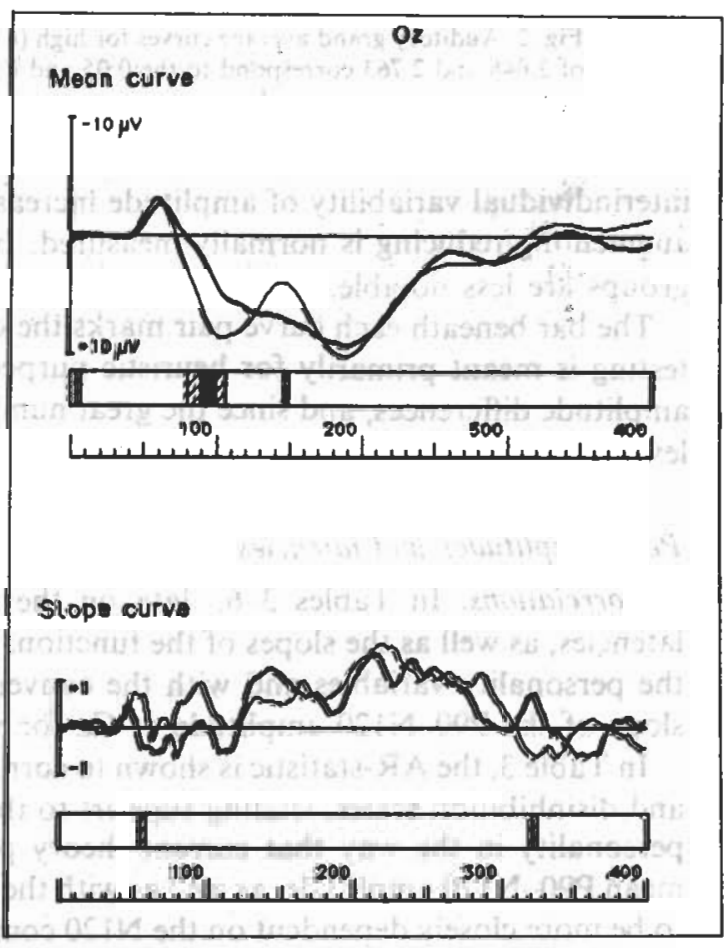

$t>2.763$

III $>2.048$

Fig. 1. Visual grand average curves for high $(n=14)$ vs low $(n=16)$ extraversion subjects. The $t$-values of 2.048 and 2.763 correspond to the 0.05 and 0.01 significance levels in single comparisons (two-tailed). 
Auditory Stimuli

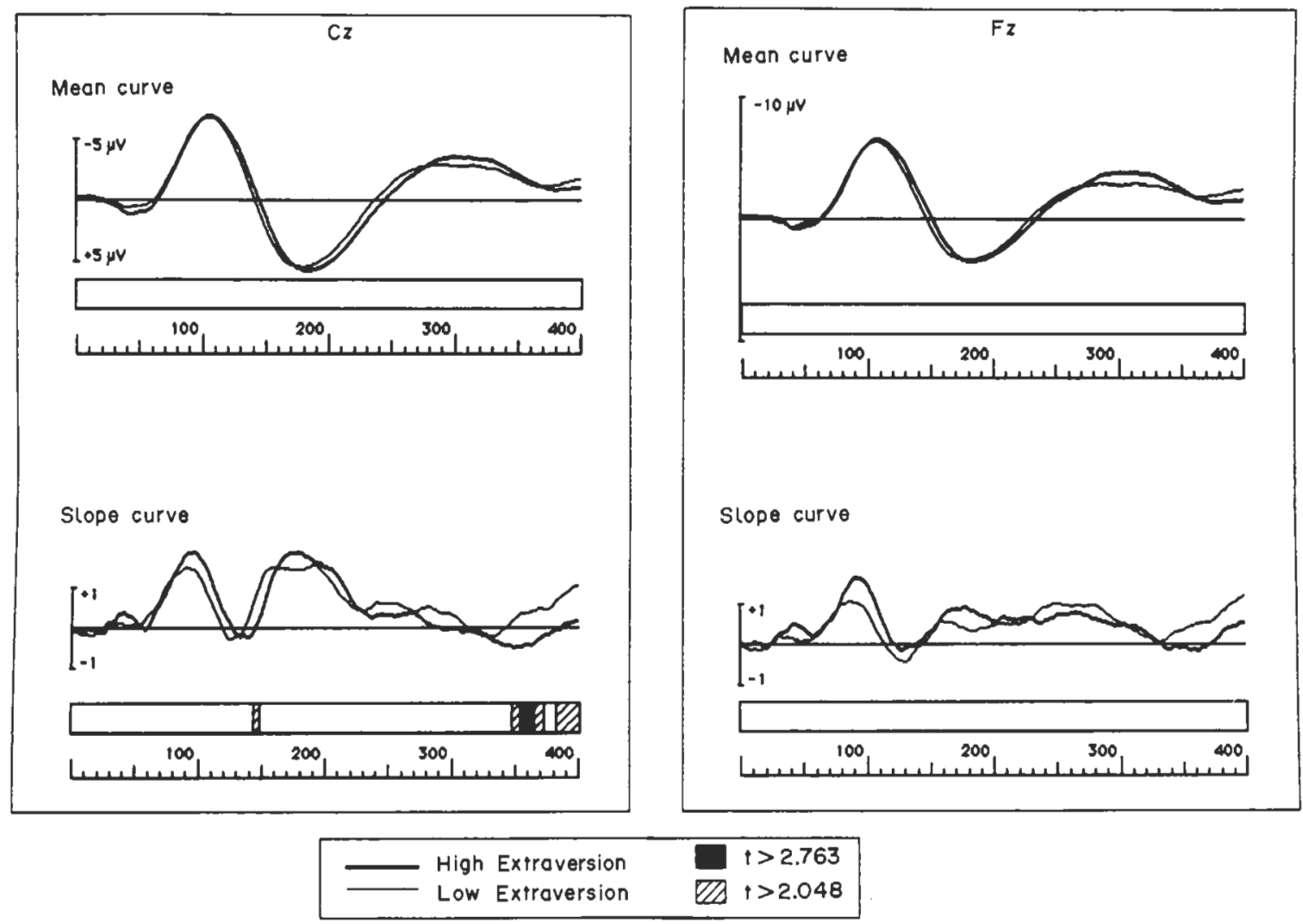

Fig. 2. Auditory grand average curves for high $(n=14)$ vs low $(n=16)$ extraversion subjects. The $t$-values of 2.048 and 2.763 correspond to the 0.05 and 0.01 significance levels in single comparisons (two-tailed).

interindividual variability of amplitude increases at the N120 peak, i.e. in the latency range where augmenting/reducing is normally measured. In the auditory EPs, differences between personality groups are less notable.

The bar beneath each curve pair marks the outcome of pairwise $t$-tests for each time point. This testing is meant primarily for heuristic purposes, since it may confound latency differences with amplitude differences, and since the great number of comparisons will compromise the significance level.

\section{Peak amplitudes and latencies}

Correlations. In Tables 3-6, data on the major components are presented. Amplitudes and latencies, as well as the slopes of the functions relating these to intensity, have been correlated with the personality variables and with the conventional augmenting/reducing (AR) measure, i.e. the slope of the P90-N120 amplitude at $\mathrm{Cz}$ for visual stimuli.

In Table 3, the AR-statistic is shown to correlate positively and significantly with the extraversion and disinhibition scales, lending support to the view that augmenting/reducing is indeed linked to personality in the way that current theory predicts. Moreover, extraversion correlates with the mean P90-N120 amplitude, as well as with the mean latency of the N120 peak. The AR effect seems to be more closely dependent on the N120 component than on the P90, and it seems to be relatively unrelated to later waves in the same curve, i.e. the P200 and the N300 (not shown in table).

In the Oz lead, (Table 4) the N60 component demonstrates interesting amplitude slope effects. Subjects scoring high on extraversion, vertex augmenting and neuroticism show clearcut reducing over intensities. No component shows amplitude changes that correspond to the vertex P90-N120 complex. The peak-to-peak amplitude most closely adjacent in time, P100-N150, exhibits an 
amplitude slope that is uncorrelated with the vertex AR statistic and a mean amplitude that correlates negatively with it. The latency measures for the early components show longer latencies for high disinhibitors.

Auditory stimuli (Tables 5 and 6) do not evoke amplitude changes in any component that relate in a consistent manner to the personality variables or the AR measure. On the whole, interindividual variations in amplitudes are less noteworthy than those in latencies. For several components, longer latencies are associated wtih extraversion, disinhibition and visual augmenting. For P60 and P200 at Fz, the latency reduction caused by higher intensities is significantly greater in extraverts.

\section{ANOVAS}

It may well be objected that slope measures take into account only linear amplitude dependencies, and that possible nonlinearities may remain unnoticed. Repeated measures analysis of variance were therefore conducted on key components, using extraversion as a grouping variable, and intensity as the repeated factor. Extraversion scores for the high and low E groups are given in Table 2. For the P90-N120 amplitude of the visual EPs at Cz, illustrated in Fig. 3, the main effects of extraversion $[F(1,28)=8.83]$ and intensity $[F(5,140)=7.93]$ were both significant (both $P<0.01$ ), as well as the interaction: extraversion $\times$ intensity $[F(5,140)=2.48, P<0.05]$.

The corresponding peak-to-peak amplitudes in the other leads (visual Oz: P100-N150, auditory $\mathrm{Cz}$ : P60-N110 and auditory Fz: P60-N110) yielded no significant effects of extraversion or extraversion $\times$ intensity. The intensity effects, however, were all significant, the strongest being the one for auditory stimuli at $\mathrm{Cz}[F(5,140)=9.79, P<0.001]$.

The ANOVAs confirm that, in comparable latency ranges, only the vertex P90-N120 amplitude for visual stimuli shows any significant augmenting/reducing related to personality.

A three-way analysis was performed on the two visual components, using extraversion as the grouping factor and electrode site and intensity as repeated factors. The result revealed a significant interaction effect of extraversion by electrode site $[F(1,28)=7.11, P=0.01]$, indicating different scalp distributions of activity in this latency range between extraversion groups. Average amplitudes for introverts were larger than those for extraverts at all intensities for the $\mathrm{Oz}$ component, whereas the reverse was true at $\mathrm{Cz}$. The interaction extraversion by intensity was also significant $[F(5,140)=2.32, P<0.05]$.

In a corresponding analysis of the two auditory components, no effects involving Extraversion came out significant.

\section{Sex effects}

The possibility of confounding effects of sex with those of extraversion was examined, first, by breaking down the high- and low-extraversion groups into male and female subgroups (Table 2). The sex distribution among extraverts was not significantly different from that among introverts $\left[\chi^{2}(1)=0.68 \mathrm{NS}\right]$. Secondly, sex was entered as a factor in a three way ANOVA of the Cz: P90-N120 data, where sex and extraversion served as grouping factors and intensity as the repeated

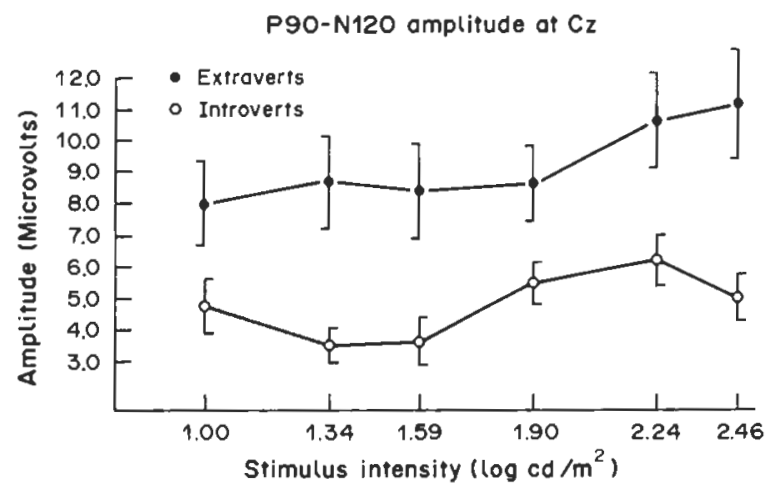

Fig. 3. Amplitudes of the P90-N120 complex at six fiash intensities. Vertical bars represent \pm 1 SE. 
factor. Neither the main effect of sex, nor the interaction sex $\times$ intensity turned out to be significant $[F(1,27)=0.11$ and $F(5,135)=1.53$, respectively $]$.

\section{DISCUSSION}

The findings of this study corroborate the view that augmenting/reducing, as traditionally defined, is related to the personality traits of extraversion and disinhibition. Subjects at the introverted and inhibited end of the personality spectrum reach their maximum amplitudes at lower intensities than others-but the conditions under which this occurs are narrowly restricted. Even with the same stimuli and at the same electrode site as the preferred definition prescribes, the phenomenon does not extend to later and larger components, which, being later, should be more important for conscious perception. In the EPs recorded over the primary visual area, no component exhibits the same behaviour as the defining vertex wave and one early negative component shows the very opposite. This is not what would be expected if reducing were a general mechanism for blunting the impact of strong stimuli. Neither does the proposed neuronal mechanism of a reticulo-cortical loop (Zuckerman et al., 1974) seem fitted for such a circumscribed effect.

The finding of contrasting relations between personality and the VEP at $\mathrm{Cz}$ and $\mathrm{Oz}$ may suggest that the personality differences reflect different ways of allocating processing resources between primary and association areas, rather than a general inhibitory mechanism.

The cross-modal comparisons further weaken the case for the sensory regulation hypothesis. According to comments from our subjects, the loudest sounds were at least as unpleasant as the brightest flashes, yet there was no consistent reducing in any group to these stimuli. Latency effects seemed more sensitive to interindividual variability than amplitude, and some of the results suggest that the size of the latency reduction caused by increasing intensities may be an alternative to amplitude augmenting/reducing.

The general tendency for extraverts and disinhibitors to display longer latencies fits well with Eysenck's theory (Eysenck, 1967) of the biological basis underlying extraversion, according to which extraverts are characterized by a lower habitual arousal level.

The failure to detect amplitude variations in the auditory EPs parallelling those in the visual, may stem from the fact that AEPs at $\mathrm{Cz}$ or $\mathrm{Fz}$ record a confluence of activity from both primary and association areas, which may obscure possible differences in processing strategy.

Acknowledgements - This study was supported by a grant (84/215-1) from the Bank of Sweden Tercentenary Foundation. We are also indebted to Associate Professor Nils Svendenius for his help with the light intensity measurement.

\section{REFERENCES}

Buchsbaum M. S. and Silverman J. (1968) Stimulus intensity control and the cortical evoked response. Psychosom. Med. 30, 12-22.

Buchsbaum M. S., Haier R. J. and Johnson J. (1983) Augmenting and reducing: individual differences in evoked potentials. In Physiological Correlates of Human Behaviour, Vol. 3 (Gale and Edwards, Eds) 117-138. Academic Press, London.

Como P. G., Simons R. and Zuckerman M. (1984) Psychophysiological indices of sensation seeking as a function of stimulus intensity. Psychophysiol. 21, 572-573.

Connolly J. F. and Gruzelier J. H. (1982) Amplitude and latency changes in the visual evoked potential to different stimulus intensities. Psychophysiol. 19, 599-608.

Eysenck H. J. (1967) The Biological Basis of Personality. Thomas, Springfield, Ill.

Eysenck H. J. and Eysenck S. B. G. (1970) Personality Structure and Measurement. Routledge (\& Kegan Paul), London. Friedman J. and Meares R. (1979) Cortical evoked potentials and extraversion. Psychosom. Med. 41, 279-286.

Haier R. J., Robinson D. L., Braden W. and Williams D. (1984) Evoked potential augmenting-reducing and personality differences. Person. individ. Diff. 5, 293-301.

Kaskey G. B., Salzman L. F., Klorman R. and Pass H. L. (1980) Relationships between stimulus intensity and amplitude of visual and auditory event related potentials. Biol. Psychol. 10, 115-125.

von Knorring L. (1974) An intra-individual comparison of pain measures, averaged evoked responses and clinical ratings during depression and after recovery. Acta Psychiat. Scand. 255, 109-120.

von Knorring L. (1980) Visual averaged evoked responses and platelet monoamine oxidase in patients suffering from alcoholism. In Biological Effects of Alcohol (Begleiter H, Ed.), pp. 649-660. Plenum Press, New York.

Mullins L. F. and Lukas J. H. (1984) Auditory augmenters are sensation seekers-if they attend the stimuli. Psychophysiol. 21, 589. 
Raine A., Mitchell D. A. and Venables P. (1981) Cortical augmenting-reducing-modality specific? Psychophysiol. 18, 700-707.

Soskis D. A. and Shagass C. (1974) Evoked potential tests of augmenting-reducing. Psychophysiol. 11, 175-190.

Zuckerman M. (1979) Sensation Seeking: Beyond the Optimal Level of Arousal. Erlbaum, Hillsdale, New Jersey.

Zuckerman M., Murtaugh T. and Siegel J. (1974) Sensation seeking and cortical augmenting/reducing. Psychophysiol. 11, $535-542$. 